

\section{La antropologia frente al Bicentenario}

\section{Anthropology in front of the Bicentennial}

Milton José Flores Chavarría

Facultad Latinoamericana de Ciencias Sociales, Ecuador

Universidad Nacional Autónoma de Nicaragua

UNAN-Managua

ID Orcid https://orcid.org/0000-0003-4301-8721

milton.flores@unan.edu.ni

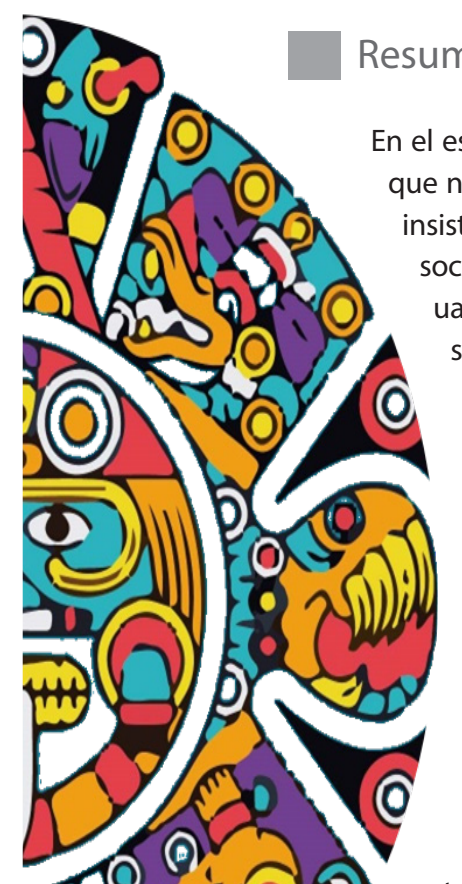

Palabras claves: antropologia, bicentenario, cultura

\section{Abstract}

In the writing resonates the great question that haunts us as Anthropologists, so our contribution will always be to assume the challenge of understanding the unifying laws, insisting on divergences and kinship. It is ours, the tradition of the study of society immersed in a culture and its historical evolution. So the proposal is to visualize: the virtue of humanism and the error of disconnection with reality (epistemic obstacle). This In Therefore, the learnings of the Bicentennial are a path with obligatory passage to admire its academic landscape: with theoretical volcanoes and lakes full of methodological approaches. That is what this invitation is about, to dare to think about the real, ideal and symbolic.

Keywords: anthropology, bicentennial, culture 


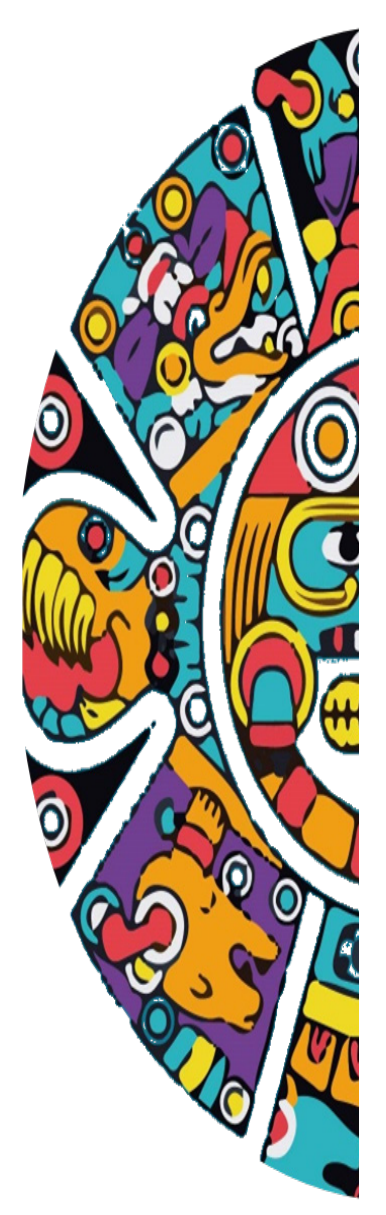

\section{Introducción}

Hace 200 años la Antropología, al menos la que conocemos, se hacía en otras latitudes. Por lo que el Bicentenario nos invita a pensarla como un desafío, al comprender una ciencia que se ha construido en este período a partir de lo situado.

En esa línea de tiempo y de ideas, es importante establecer que han transcurrido casi tres décadas desde la fundación de la carrera, de la mano de profesionales que la instituyeron como formación disciplinaria en el seno de la Universidad Nacional Autónoma de Nicaragua (López, et al., 2017; Alvarado, 2019). Sin embargo, es de vieja data que la Antropología y sus sub-disciplinas (física, arqueológica, lingüística, etnohistoria, social y etnológica), expresan sus capacidades para exponer y desenvolver la reflexión teórica entorno a la actividad académica nicaragüense.

Es por ello que es importante destacar la capacidad de pensar nuestro quehacer con la amplitud heurística, donde converge la antropología: cultural, económica, jurídica, política y urbana. Ya que, de esta manera se constituyó una modelo particular de concebir y hacer disciplina a partir de la interdisciplinariedad con la que se originó; lo cual, creó una comunidad antropológica particular de la que participamos y somos herederos. Lo que a su vez nos otorga identidad, un sello propio que nos distingue dentro del escenario nacional, regional y latinoamericano. Sin duda, esta peculiar característica de las antropólogas y antropólogos de Nicaragua es la que nos interesa exponer en el contexto del Bicentenario.

\section{Desarrollo}

Por lo tanto, esta efeméride motiva el repensarnos de acuerdo a lo que hacemos como sentipensantes (Galeano, 1989), ya que de tanto caminar con el otro, el humanismo florece en la piel, acompañada por emociones, sentimientos que crean y se recrean en el vaivén (retroalimentación) entre teorías y conceptos de nuestro quehacer antropológico y su cargada tradición científica; dos elementos que nos llevan a pensar a la Antropología nicaragüense como una identidad proyecto. Me refiero en primer lugar, a enfrentarnos a lo clásico, a esos que piensan lo cualitativo como pura especulación porque solo miden.

De esta manera el segundo punto, recae en la importancia de la etnografía como estrategia donde la realidad del contexto, los conocimientos (saberes, teorías y métodos) y las actitudes son parte del todo complejo, pero solo encuentran sentido cuando cuidamos nuestra capacidad de asombro (Krotz, 2002) y vigilancia epistémica (Bourdieu, et al., 1973).

Me refiero, que de tanto andar con el otro nos colocamos en sus zapatos, usamos sus sandalias [SIC: chinelas] o las bota de hule [SIC: botadihule], luego nos colocamos los de investigadores/as, para solo así, unirnos a la esencia bípeda de caminar descalzos como seres humanos. 
Es ahí donde simbólicamente nos abrazamos, esa es la mismisidad que otros niegan (Alteridad), también es el encuentro subjetividad-objetividad en lo vivido. Sujeto, objeto e investigador/a: triangulando, conjeturando, refutando y reacomodándose.

Resulta claro que el error más común en estos 200 años, encuentra una explicación más sostenida en el refrán popular "a la fuerza ni los zapatos". Se trata de una triada hegeliana y por supuesto marxista entre: Realidad, Teoría y Método. Donde como nicaragüenses no nos hemos analizado, más bien, privilegiamos a la teoría o al método antes que a la realidad posible del lugar. Por eso, desde la investigación social seguimos pensando, y por ende, identificando al sujeto bajo preceptos, métodos y teorías (inter y transdisciplinarias) que no se apegan a su entorno cultural profundo.

De esa forma, seguimos tendencias descontextualizadas, por ejemplo, al llamar en afrodescendientes cuando nos referirnos a los Estudios de la Negritud. También cuando afirmamos que por ubicarse en zonas rurales nos abocamos con poblaciones campesinas. $Y$ en última instancia, cuando identificamos infantes que trabajan en la economía familiar, nos colocamos de frente al concepto de deserción escolar.

En ese tenor, como resultado se genera una reacción como si nos negáramos al cambio, por una voz que retumba en el inconsciente colectivo "así como está, está bien" ya que de la otra forma, aparenta ser compleja; no por difícil, sino por ser multifactorial. Entonces, se vuelve necesario aceptar que es rara, diferente, una realidad atípica, una ruralidad diversa y concreta, con resultados comunes, pero con sorpresas: sujetos rurales sin agricultura, que no conocen de animales, de lunas o de medicinas de tradicionales.

Ese es el resultado del error heredado de occidente, un obstáculo epistémico, tan interiorizado e institucionalizado (bio-psico-sociocultural y espiritualmente) por los mismos que nos mostraron al indígena en la época colonial como: el atraso, inferior, salvaje, bárbaro; para luego transferir su carga simbólica al imaginario de lo rural. Varios elementos que se reúnen en lo que se le denominó como proceso civilizatorio, al mismo tenor que el de evangelización, por eso "hay que mejorar la raza", en una visión de progreso, pero que en realidad, esconde muchos silencios representados en el no poder soltar el pasado a placer, ya que nos delata nuestro gusto rural de las cosas.

Así, entre ensayo y error, después de varias décadas, hay un nuevo contexto nacional e intelectual donde nuestra comunidad se consolida en este Departamento de Antropología. Frente a esta situación, retomamos un llamado de integración, crecimiento y renovación, pero también de debate y autocrítica, con base a nuestra historia que se configura en esta casa de estudios, sin duda la más importante del país.

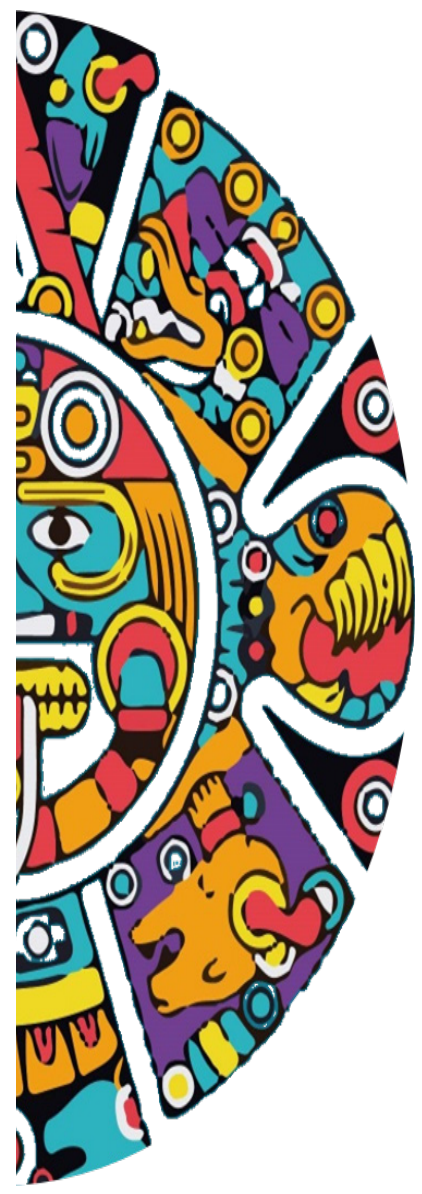




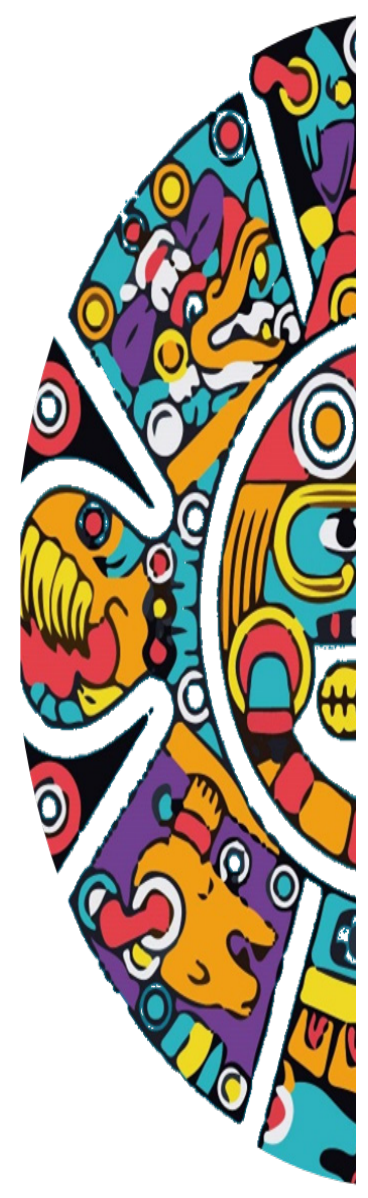

No obstante, todavía debemos hacer el ejercicio de cómo pasamos de ser indios y españoles, a criollos y campesinos, inmigrantes urbanos y ciudadanos, del dominio masculino a la horizontalidad con lo femenino, reconocer el desarrollo económico, pero también todas las desigualdades que conlleva su parte discursiva. De esa forma comprender el regalo que nos brindan elementos para recrearnos en una sociedad más justa y equitativa, por eso, comprobado lo tenemos, que el amor a la paz, siempre será el camino.

\section{Conclusión}

En ese sentido, es importante preguntarnos si estos dos siglos será una celebración general, porque si lo analizamos a partir del otro, que es la gracia de la Antropología; se hace necesario profundizar en las comunidades indígenas y creoles, los grupos vulnerables o para quienes son parte de las diversidades sociales. Entonces, a partir de las otras realidades, significa que hemos constituido un estado que reconoce esas identidades en vilo.

Para prueba, tenemos varios botones, hoy ya constitucionales, no obstante me gustaría centrarme en la historia reciente que nos recuerda: la dictadura de Somoza, los tres gobiernos neoliberales de capitalismo salvaje y la barbarie de la extrema derecha en 2018. Estos tres momentos históricos son indicadores inequívocos que debemos celebrar lo que tanto nos cuestan estos 200 años. Y son en estas áreas donde nuestra Antropología tiene una grande deuda, pero a su vez, es alentadora para enfrentar un tercer siglo. 


\section{Bibliografía}

Alvarado, A. (2019). Investigaciones antropológicas indispensables en el desarrollo de los pueblos. Reportaje. En Artículos, entrevistas y reportajes. Unan-Managua. Disponible en https://www.unan.edu.ni/index.php/articulos-entrevistas-reportajes/investigaciones-antropologicas-indispensables-en-el-desarrollo-de-los-pueblos.odp

Bonfil-Batalla, G. (1981). Lo propio y lo ajeno: una aproximación al problema del control cultural. Revista Mexicana de Ciencias Políticas y Sociales. Vol. 27. №. 103. pp. 183-191. Disponible en http://www.revistas.unam.mx/index. php/rmcpys/article/view/72329

Bourdieu, P., Chamboredon, J. y Passeron, J. (1973). El oficio de sociólogo. Siglo XXI: México.

Galeano, E. (1989). El libro de los abrazos. Siglo XXI: Argentina. Disponible en http://resistir.info/livros/galeano_el_libro_de_los_abrazos.pdf

Krotz, E. (2002). La otredad cultural entre utopía y ciencia. Un estudio sobre el origen, el desarrollo y la reorientación de la antropología. Fondo de Cultura Económica/Universidad Autónoma Metropolitana-Iztapalapa: México.

López, G.; Andino, E. y Álvarez, M. (2017). Veinte años de Antropología en Nicaragua. Revista Nicaragüense de Antropología - Raíces. №1. Vol. 1. pp. 15-26. Disponible en https://repositoriosiidca.csuca.org/Record/RepoUNANM11830/Details

Milton José Flores Chavarría

Maestrante Estudios Socioambientales (Ambiente, desarrollo y territorio), Facultad Latinoamericana de Ciencias Sociales, Ecuador. Maestrante (Facultad de Filosofía y Letras/Instituto de Investigaciones Antropológicas) Universidad Nacional Autónoma de México Biblioteca Nacional de México, Profesor/Investigador (Antropología) Universidad Nacional Autónoma de Nicaragua. Interesado en la investigación y la docencia lo ha llevado como Antropólogo a la transdisciplinariedad. De esta forma amplió su panorama de estudios socioculturales entre las temáticas: Desarrollo, Ambiente y Territorio. A partir del enfoque etnográfico como productor de sentido y compresión cultural en contexto de globalización.

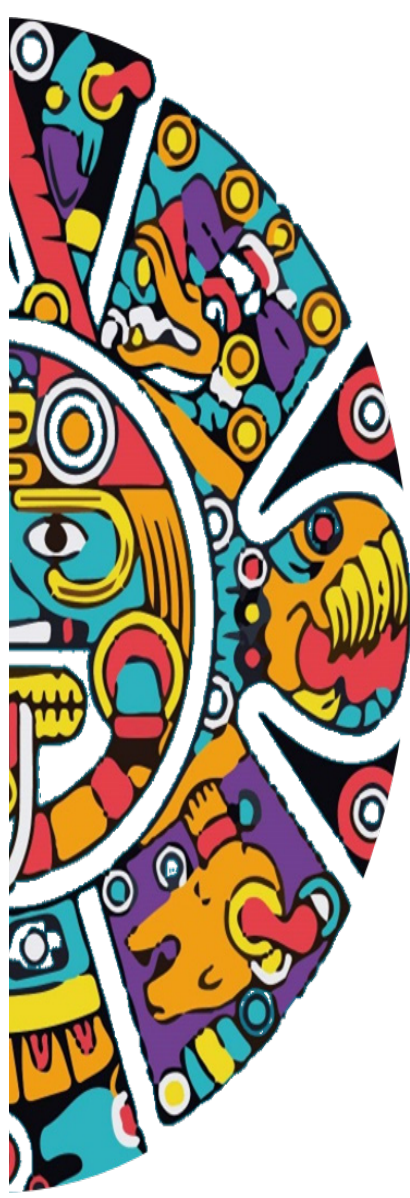

